\title{
EXISTENCE RESULTS FOR SEMILINEAR ELLIPTIC EQUATIONS WITH SMALL MEASURE DATA
}

\author{
Nathalie GRENON \\ Faculté des Sciences de Bourges, rue Gaston Berger B.P. 4043, 18028 Bourges Cedex, France \\ Received 13 June 2000, revised 5 March 2001
}

ABSTRACT. - We give a smallness condition on $|m|$, and $\|f\|_{q}$ for the existence of a solution for the model problem: $-\Delta_{p} u=f(x)|u|^{\gamma}+m \mu$ with $u=0$ on $\partial \Omega$, where $\Omega$ is a bounded open set of $\mathbb{R}^{N}, f(x) \in L^{q}(\Omega), q \geqslant 1, m \in \mathbb{R}$ and $\mu$ is a Radon measure with bounded variation on $\Omega$ such that $|\mu|(\Omega)=1$.

(C) 2002 L'Association Publications de l'Institut Henri Poincaré. Published by Elsevier B.V. All rights reserved

RÉSUMÉ. - Nous donnons une condition suffisante sur $|m|$, et $\|f\|_{q}$ pour l'existence de solution au problème modèle : $-\Delta_{p} u=f(x)|u|^{\gamma}+m \mu$ avec $u=0$ sur $\partial \Omega$, où $\Omega$ est un ouvert borné de $\mathbb{R}^{N}, f(x) \in L^{q}(\Omega), q \geqslant 1, m \in \mathbb{R}$ et $\mu$ est une mesure de Radon à variation bornée sur $\Omega$ telle que $|\mu(\Omega)|=1$.

(C) 2002 L'Association Publications de l'Institut Henri Poincaré. Published by Elsevier B.V. All rights reserved

\section{Introduction and main results}

The main goal of this paper is to prove, if the data are small enough, the existence of a solution for the model problem

$$
\begin{cases}-\Delta_{p} u=f(x)|u|^{\gamma}+m \mu & \text { in } \Omega, \\ u=0 & \text { on } \partial \Omega,\end{cases}
$$

where $N \geqslant 1, \Omega$ is a bounded open subset of $\mathbb{R}^{N},-\Delta_{p}$ is the so called $p$-Laplace operator, $f(x) \in L^{q}(\Omega), q \geqslant 1, \mu \in M_{B}(\Omega)$ (that is to say $\mu$ is a Radon measure with bounded variation in $\Omega$ ) such that $|\mu|(\Omega)=1$ and $m \in \mathbb{R}$.

In fact we study the more general problem

$$
\begin{cases}-\operatorname{div}(a(x, D u))=h(x, u)+m \mu & \text { in } \Omega \\ u=0 & \text { on } \partial \Omega\end{cases}
$$

where $u \mapsto-\operatorname{div}(a(x, D u))$ is a monotone operator defined on $W_{0}^{1, p}(\Omega)$ with values in $W^{-1, p^{\prime}}(\Omega), p>1, \frac{1}{p}+\frac{1}{p^{\prime}}=1$. We suppose more precisely that,

$$
a: \Omega \times \mathbb{R}^{N} \rightarrow \mathbb{R}^{N} \text { is a Caratheodory function, }
$$

E-mail address: Nathalie.Grenon@ univ-Orleans.fr (N. Grenon). 
that is to say $a(., \xi)$ is measurable on $\Omega$ for every $\xi$ in $\mathbb{R}^{N}$, and $a(x,$.$) is continuous on$ $\mathbb{R}^{N}$ for almost every $x$ in $\Omega$, that,

$$
a(x, \xi) \xi \geqslant \alpha|\xi|^{p}
$$

for almost every $x$ in $\Omega$ and for every $\xi$ in $\mathbb{R}^{N}$, where $\alpha>0$ is a constant, that,

$$
|a(x, \xi)| \leqslant d(b(x)+|\xi|)^{p-1},
$$

for almost every $x$ in $\Omega$ and every $\xi$ in $\mathbb{R}^{N}$, where $d>0$ is a constant and $b$ is a nonnegative function in $L^{p}(\Omega)$, and that,

$$
\left(a(x, \xi)-a\left(x, \xi^{\prime}\right)\right)\left(\xi-\xi^{\prime}\right)>0,
$$

for almost $x$ in $\Omega$, and for every $\xi, \xi^{\prime}$ in $\mathbb{R}^{N}, \xi \neq \xi^{\prime}$. We also assume that,

$$
h: \Omega \times \mathbb{R} \rightarrow \mathbb{R} \text { is a Caratheodory function, }
$$

that is to say $h(., t)$ is measurable on $\Omega$ for every $t$ in $\mathbb{R}$, and $h(x,$.$) is continuous on \mathbb{R}$ for almost every $x$ in $\Omega$, and that,

$$
\left\{\begin{array}{l}
|h(x, t)| \leqslant f(x)|t|^{\gamma} \\
\text { for some } 1 \leqslant \gamma<+\infty \text { and some } f \in L^{q}(\Omega), \\
\text { where } 1 \leqslant q \leqslant+\infty
\end{array}\right.
$$

for almost every $x$ in $\Omega$ for every $t$ in $\mathbb{R}$.

Observe that there is no sign assumption on $h(x, t)$, only the growth on $t$ is considered.

We now recall some well known results about measures.

For every measure $\mu \in M_{B}(\Omega)$ there exists a unique pair of measures $\left(\mu_{0}, \mu_{s}\right)$ such that $\mu=\mu_{0}+\mu_{s}$ (see [5] and [10]) with $\mu_{0}$ in $M_{0}(\Omega)$ (that is to say the set of all measures in $M_{B}(\Omega)$ which are absolutely continuous with respect to the $p$-capacity) and $\mu_{s}$ in $M_{S}(\Omega)$ (that is to say the set of all measures in $M_{B}(\Omega)$ which are singular with the $p$-capacity). In other words, $\mu_{s}$ is concentrated on a subset $E$ of $\Omega$ with zero $p$-capacity, and $\mu_{0}$ does not charge the set of zero $p$-capacity. Moreover it is equivalent for a measure to be in $M_{0}(\Omega)$ and to belong to $L^{1}(\Omega)+W^{-1, p^{\prime}}(\Omega)$, that is to say every $\mu_{0}$ can be written as $\mu_{0}=f-\operatorname{div} g$ with $f \in L^{1}(\Omega)$ and $g \in\left(L^{p^{\prime}}(\Omega)\right)^{N}$. In short, every $\mu \in M_{B}(\Omega)$ can be decomposed as follows,

$$
\mu=f-\operatorname{div} g+\mu_{s}^{+}-\mu_{s}^{-}
$$

where $f \in L^{1}(\Omega), g \in\left(L^{p^{\prime}}(\Omega)\right)^{N}, \mu_{s}^{+}, \mu_{s}^{-}$(the positive part and negative part of $\mu_{s}$ ) are two nonnegative measures in $M_{s}(\Omega)$ which are concentrated on two disjoint subsets $E^{+}$and $E^{-}$of zero $p$-capacity. Recall also (see $[3,7,8]$ ) that if $u$ is a measurable function defined on $\Omega$, which is finite almost everywhere, and satisfies $T_{k}(u) \in W_{0}^{1, p}(\Omega)$ for every $k>0$ (where $T_{k}(u)$ is the truncate at level $k$ ), then there exists a measurable function $v: \Omega \rightarrow \mathbb{R}^{N}$ such that $D T_{k}(u)=v \chi_{\{|u| \leqslant k\}}$ almost everywhere in $\Omega$, for every $k>0$, 
which is unique up to almost everywhere equivalence. We define the gradient $D u$ of $u$ as this function $v$.

Let us recall the definition of a renormalized solution (see [7,8]).

Definition 1.1. - We suppose (1.3)-(1.6), $p>1, \mu \in M_{B}(\Omega)$. We say that $u$ is a renormalized solution of

$$
\begin{cases}-\operatorname{div}(a(x, D u))=\mu & \text { in } \Omega, \\ u=0 & \text { on } \partial \Omega\end{cases}
$$

if,

- the function $u$ is measurable and finite everywhere and $T_{k}(u)$ belongs to $W_{0}^{1, p}(\Omega)$ for every $k>0$,

- the gradient Du in the previous sense satisfies,

$$
|D u|^{p-1} \in L^{q}(\Omega), \quad \forall q, 1 \leqslant q<\frac{N}{N-1},
$$

- if $w$ belongs to $W_{0}^{1, p}(\Omega) \cap L^{\infty}(\Omega)$ and if there exists $k>0$ and $w^{+\infty}, w^{-\infty} \in$ $W^{1, r}(\Omega) \cap L^{\infty}(\Omega)$ with $r>N$ such that,

$$
\begin{gathered}
w=w^{+\infty} \quad \text { a.e. on the set }\{u>k\}, \\
w=w^{-\infty} \quad \text { a.e. on the set }\{u<-k\},
\end{gathered}
$$

then,

$$
\int_{\Omega} a(x, D u) D w \mathrm{~d} x=\int_{\Omega} w \mathrm{~d} \mu_{0}+\int_{\Omega} w^{+\infty} \mathrm{d} \mu_{s}^{+}-\int_{\Omega} w^{-\infty} \mathrm{d} \mu_{s}^{-} .
$$

In [8] the authors give equivalent definitions of renormalized solutions. When $\mu \in$ $M_{0}(\Omega)$, this definition is equivalent to the definition of an entropy solution (see [3] and [5]).

Let us observe that when $p>N$, the renormalized solution is just a usual weak solution and belongs to some $C^{0, \alpha}(\Omega)$; therefore the notion of renormalized solution is not really needed. This is also the case for example in the linear case where $a(x, \xi)=$ $A(x) \xi$ when the matrix $A$ has smooth coefficients. However, when the coefficients are not smooth, a new notion is necessary even in the linear case in order to obtain both existence and uniqueness results (see [16]). Observe in particular that the test function $w$ which is used in (1.10) actually depends on the solution $u$ itself, and that in some sense $u=+\infty$ on the set where $\mu_{s}^{+}$is concentrated, while $u=-\infty$ on the set where $\mu_{s}^{-}$is concentrated since the action of $\mu_{s}$ on the set where $|u| \leqslant k$ does not appear in (1.10). For more comments on the notion of renormalized solutions, see [8]. These equations have been widely studied. Especially in $[1,2,11]$, the authors give a sufficient and necessary condition for the existence of a solution of equations closed to (1.2) in the case $p=2$, but their method doesn't extend to $p \neq 2$. See also [15] for the case of an 
eigenvalue problem. Let us also quote [4] in which the authors give counter examples to the existence for the equation of the type (1.2). Quasilinear equations have been studied with more regular data in $[9,12,14]$ for instance. In these papers existence results are obtained assuming that the data are small enough relatively to a convenient norm.

The main result of this paper is the following,

THEOREM 1.1. - Assume (1.3)-(1.8), let $m \in \mathbb{R}$ and $\mu \in M_{B}(\Omega)$, such that $|\mu|(\Omega)=1,1 \leqslant \gamma<+\infty, 1 \leqslant q \leqslant+\infty$ with $q \neq 1$ if $N=p$ and $\gamma q^{\prime}<\frac{(p-1) N}{N-p}$ if $N>p$. Then there exists a renormalized solution of (1.2)

(1) if $1 \leqslant \gamma<p-1$ (thus $p>2$ )

with no additionnal condition on $\|f\|_{q}, m$;

(1) if $\gamma \geqslant p-1$ then the condition is

$$
\|f\|_{q}|m|^{\frac{\gamma-p+1}{p-1}} \leqslant \frac{C}{|\Omega|^{\frac{1}{q}+\frac{\gamma}{p-1}\left(-1+\frac{p}{N}\right)}}
$$

for some constant $C=C(N, p, \gamma)$.

Remarks. -

- First observe that when $p<N$, there exists some $q$ with $1 \leqslant q \leqslant+\infty$ and some $\gamma \geqslant 1$ such that $\gamma q^{\prime}<\frac{(p-1) N}{N-p}$ if and only if $p>\frac{2 N}{N+1}$.

This is a restriction on the values of $\gamma$ and $q$, which is natural. Indeed, in order to define a renormalized solution of (1.2), we need $h(x, u)$ to belong to $L^{1}(\Omega)$. But even if $h(x, u) \equiv 0$, the renormalized solution $u$ of (1.2) belongs to $L^{r}(\Omega)$ for any $r, 1 \leqslant r<\frac{(p-1) N}{N-p}$ and is not in general in $L^{\frac{(p-1) N}{N-p}}(\Omega)$. Consequently if $\gamma q^{\prime} \geqslant \frac{(p-1) N}{N-p}$ we shall not have $h(x, u) \in L^{1}(\Omega)$.

- If $\gamma=p-1$ condition (1.11) reads

$$
\|f\|_{q} \leqslant C|\Omega|^{\frac{1}{q}-\frac{p}{N}}
$$

with no condition on $m$. Actually, if $u$ solves

$$
-\Delta_{p} u=f(x)|u|^{p-1}+m \mu,
$$

then for any $c>0, v=c u$ solves

$$
-\Delta_{p} v=f(x)|v|^{p-1}+c^{p-1} m \mu .
$$

That is to say, if there is a solution for $m$ and $\mu$ given, then there is a solution for every $|m|$.

- If $\mu \geqslant 0$ and $h \geqslant 0$, then a solution of (1.2) is nonnegative. Indeed, we can use $w=-T_{k}\left(u^{-}\right)$as test function in the equation satisfied by $u$ and then (observe that $\mu_{s}^{-}=0$ and $\left.w^{+\infty}=0\right)$

$$
-\int_{\Omega} a(x, D u) D T_{k}\left(u^{-}\right) \mathrm{d} x=\int_{\Omega} h(x, u)\left(-T_{k}\left(u^{-}\right)\right) \mathrm{d} x+\int_{\Omega}-T_{k}\left(u^{-}\right) \mathrm{d} \mu_{0} \leqslant 0,
$$


from (1.4), we deduce that,

$$
\alpha\left\|D T_{k}\left(u^{-}\right)\right\|_{p} \leqslant 0
$$

for any $k>0$, and then $u^{-}=0$. It means that Theorem 1.1 gives conditions for the existence of a positive renormalized solution of

$$
\begin{cases}-\Delta_{p} u=h(x, u)+\mu & \text { in } \Omega \\ u=0 & \text { on } \partial \Omega\end{cases}
$$

\section{Estimates and preliminary lemmas}

Recall the following estimates,

LEMMA 2.1. - We suppose (1.3)-(1.6), $\mu \in M_{B}(\Omega)$, such that $|\mu|(\Omega)=1, m \in \mathbb{R}$ and $p>1$. Let $u$ be a renormalized solution of

$$
\begin{cases}-\operatorname{div}(a(x, D u))=m \mu & \text { in } \Omega, \\ u=0 & \text { on } \partial \Omega\end{cases}
$$

then the following estimate holds

$$
\|u\|_{r} \leqslant C|\Omega|^{\frac{1}{r}+\frac{1}{p-1}\left(-1+\frac{p}{N}\right)}|m|^{\frac{1}{p-1}},
$$

for some positive constant $C=C(N, p, r)$ and for any $r \in[1,+\infty]$ if $p>N, r \in$ $[1,+\infty)$ if $p=N$, and $r \in\left[1, \frac{N(p-1)}{N-p}\right)$ if $p<N$.

This estimate is proven in [13] for instance, where explicit value for $C$ is explicitely given in a more general context. It can also be proven by symmetrization techniques (see [17]). We have to specify that in [13], the right-hand side is in $L^{1}(\Omega)$, but the proof extends to $\mu \in M_{B}(\Omega)$ without difficulty.

COROllary 2.1. - Assume (1.3)-(1.8), $1 \leqslant \gamma<+\infty, 1 \leqslant q \leqslant+\infty$. If $v \in L^{\gamma q^{\prime}}(\Omega)$, $m \in \mathbb{R}$ and $\mu \in M_{B}(\Omega)$ such that $|\mu|(\Omega)=1$, if $q \neq 1$ when $N=p$ and if $\gamma q^{\prime}<\frac{(p-1) N}{N-p}$ (thus $p>\frac{2 N}{N+1}$ ) when $N>p$, and if $u$ is a renormalized solution of

$$
\begin{cases}-\operatorname{div}(a(x, D u))=h(x, v)+m \mu & \text { in } \Omega, \\ u=0 & \text { on } \partial \Omega\end{cases}
$$

then,

$$
\|u\|_{\gamma q^{\prime}} \leqslant A+B\|v\|_{\gamma q^{\prime}}^{\frac{\gamma}{p-1}}
$$

where

$$
A=C|\Omega|^{\frac{1}{\gamma q^{\prime}}+\frac{1}{p-1}\left(-1+\frac{p}{N}\right)}|m|^{\frac{1}{p-1}}, \quad B=C|\Omega|^{\frac{1}{\gamma q^{\prime}}+\frac{1}{p-1}\left(-1+\frac{p}{N}\right)}\|f\|_{q}^{\frac{1}{p-1}}
$$

for some positive constant $C=C(N, p, \gamma)$. 
Proof. - We have

$$
(|h(x, v)+m \mu|(\Omega))^{\frac{1}{p-1}} \leqslant\left(\|h(x, v)\|_{1}+|m|\right)^{\frac{1}{p-1}}
$$

then from (1.8), and Hölder inequality,

$$
(|h(x, v)+m \mu|(\Omega))^{\frac{1}{p-1}} \leqslant\left(\|v\|_{\gamma q^{\prime}}^{\gamma}\|f\|_{q}+|m|\right)^{\frac{1}{p-1}}
$$

and then,

- if $\frac{1}{p-1}<1$,

$$
(|h(x, v)+m \mu|(\Omega))^{\frac{1}{p-1}} \leqslant\|f(x)\|_{q}^{\frac{1}{p-1}}\|v\|_{\gamma q^{\prime}}^{\frac{\gamma}{p-1}}+|m|^{\frac{1}{p-1}},
$$

- if $\frac{1}{p-1} \geqslant 1$,

$$
(|h(x, v)+m \mu|(\Omega))^{\frac{1}{p-1}} \leqslant 2^{\frac{2-p}{p-1}}\|f(x)\|_{q}^{\frac{1}{p-1}}\|v\|_{\gamma q^{\prime}}^{\frac{\gamma}{p-1}}+2^{\frac{2-p}{p-1}}|m|^{\frac{1}{p-1}}
$$

and we get the corollary from (2.1) with $r=\gamma q^{\prime}$.

We now study the function, $\varphi: \mathbb{R}^{+} \rightarrow \mathbb{R}$ defined by,

$$
\varphi(X)=A+B X^{\frac{\gamma}{p-1}}-X
$$

where $A, B \geqslant 0$.

- If $\gamma>p-1$, then, $\varphi(0)=A \geqslant 0$ and $\lim _{X \rightarrow+\infty} \varphi(X)=+\infty$, moreover, by calculation of the derivative, we get that $\varphi$ has a minimum at the point,

$$
X_{0}=\left(\frac{p-1}{B \gamma}\right)^{\frac{p-1}{\gamma-p+1}}
$$

with

$$
\varphi\left(X_{0}\right)=A+\frac{1}{\gamma^{\frac{\gamma}{\gamma-p+1}}} \frac{(p-1)^{\frac{p-1}{\gamma-p+1}}}{B^{\frac{p-1}{\gamma-p+1}}}(p-1-\gamma),
$$

then $\varphi$ has at least one root if and only if $\varphi\left(X_{0}\right) \leqslant 0$ that is to say if,

$$
A B^{\frac{p-1}{\gamma-p+1}} \leqslant \frac{1}{\gamma^{\frac{\gamma}{\gamma-p+1}}}(p-1)^{\frac{p-1}{\gamma-p+1}}(\gamma+1-p),
$$

and $\varphi$ has two roots if,

$$
A B^{\frac{p-1}{\gamma-p+1}}<\frac{1}{\gamma^{\frac{\gamma}{\gamma-p+1}}}(p-1)^{\frac{p-1}{\gamma-p+1}}(\gamma+1-p) .
$$


- If $\gamma=p-1$, then,

$$
\varphi(X)=(B-1) X+A,
$$

then $\varphi$ has a root if

$$
B<1, \quad \forall A \geqslant 0 .
$$

- If $\gamma<p-1$, then,

$$
\varphi(X)=A+B X^{\frac{\gamma}{p-1}}-X
$$

and then,

$$
\lim _{X \rightarrow+\infty} \varphi(X)=-\infty \quad \text { and } \quad \varphi(0) \geqslant 0,
$$

then $\varphi$ has a root for any $A, B \geqslant 0$.

We henceforth denote (when it exists),

$$
Y \text { : the smallest root of } \varphi \text {. }
$$

\section{Proof of Theorem 1.1}

First observe that,

- if $\gamma>p-1$, condition (2.3) is equivalent to

$$
|\Omega|^{\frac{1}{q^{\prime}}+\frac{\gamma}{p-1}\left(-1+\frac{p}{N}\right)}|m|^{\frac{\gamma-p+1}{p-1}}\|f(x)\|_{q} \leqslant C
$$

for some constant $C=C(N, p, \gamma)$.

- if $\gamma=p-1$, condition (2.4) is equivalent to

$$
|\Omega|^{-\frac{1}{q}+\frac{p}{N}}\|f(x)\|_{q} \leqslant C
$$

for some constant $C=C(N, p)$, and we recognize the condition which appear in the second case of Theorem 1.1.

We set

$$
h_{n}(s)=T_{n}(h(s)),
$$

where $T_{n}$ is the truncate at level $n$.

Lemma 3.1. - We suppose (1.3)-(1.8), let $\mu \in M_{B}(\Omega) \cap W^{-1, p^{\prime}}(\Omega)$, such that $|\mu|(\Omega)=1$ and $m \in \mathbb{R}$, we suppose that $Y$ defined by (2.5) exists, that is to say if the previous conditions are fulfilled. Then, for any $\mu_{n} \in W^{-1, p^{\prime}}(\Omega) \cap M_{B}(\Omega)$ such that, $\left|\mu_{n}\right|(\Omega) \leqslant m$ there exists a solution $u \in W_{0}^{1, p}(\Omega)$ of the equation:

$$
\left\{\begin{array}{l}
\int_{\Omega} a(x, D u) D w \mathrm{~d} x=\int_{\Omega} h_{n}(x, u) w \mathrm{~d} x+\left\langle\mu_{n}, w\right\rangle \\
\forall w \in W_{0}^{1, p}(\Omega),
\end{array}\right.
$$


such that,

$$
\|u\|_{\gamma q^{\prime}} \leqslant Y,
$$

where $\gamma, q^{\prime}$ satisfy the same conditions as in Corollary 2.1.

Proof. - We shall use Schauder Fixed Point Theorem.

Let $v \in W_{0}^{1, p}(\Omega)$ then $h_{n}(x, v)+\mu_{n} \in W^{-1, p^{\prime}}(\Omega)$ and there exists a unique $u \in$ $W_{0}^{1, p}(\Omega)$, such that,

$$
\left\{\begin{array}{l}
\int_{\Omega} a(x, D u) D w \mathrm{~d} x=\int_{\Omega} h_{n}(x, v) w \mathrm{~d} x+\left\langle\mu_{n}, w\right\rangle \\
\forall w \in W_{0}^{1, p}(\Omega) .
\end{array}\right.
$$

Moreover since $\left|h_{n}(v)\right| \leqslant n$, using $u$ as test function we easily get

$$
\|D u\|_{p} \leqslant C_{n},
$$

where $C_{n}$ is a constant which depends on $n$ but not on $v$.

Let $v \in W_{0}^{1, p}(\Omega)$, we henceforth set $A_{n}(v)=u$ the solution of (3.2). Let $E=\left\{v \in W_{0}^{1, p}(\Omega) \cap L^{\gamma q^{\prime}}(\Omega),\|D v\|_{p} \leqslant C_{n},\|v\|_{\gamma q^{\prime}} \leqslant Y\right\}$, then,

- $E$ is a closed convex subset of $W_{0}^{1, p}(\Omega)$.

- Observe that from definition of $Y$, if $v \in E$ then

$$
\|u\|_{\gamma} \leqslant A+B\|v\|_{\gamma}^{\frac{\gamma}{p-1}} \leqslant A+B Y^{\frac{\gamma}{p-1}}=Y .
$$

Moreover we have already seen that

$$
\|D u\|_{p} \leqslant C_{n}
$$

then,

$$
A_{n}: E \rightarrow E \text {. }
$$

- Suppose that $\left(v_{\varepsilon}\right)$ is a sequence in $E$ such that $v_{\varepsilon} \rightarrow v$ in $W_{0}^{1, p}(\Omega)$ strong and let $u_{\varepsilon}=A\left(v_{\varepsilon}\right)$. Since $\left(v_{\varepsilon}\right)$ is bounded in $W_{0}^{1, p}(\Omega)$ there exists a subsequence still denoted $\left(u_{\varepsilon}\right)$ such that,

$$
u_{\varepsilon} \rightarrow u L^{p}(\Omega) \text { strong, a.e. in } \Omega \text { and } W_{0}^{1, p}(\Omega) \text { weak. }
$$

Using $\left(u_{\varepsilon}-u\right)$ as test function in (3.2) we get,

$$
\int_{\Omega} a\left(x, D u_{\varepsilon}\right) D\left(u_{\varepsilon}-u\right) \mathrm{d} x=\int_{\Omega} h_{n}\left(v_{\varepsilon}\right)\left(u_{\varepsilon}-u\right) \mathrm{d} x+\left\langle\mu_{n}, u_{\varepsilon}-u\right\rangle .
$$

We can easily see that the right-hand side tends to zero as $\varepsilon$ tends to zero, then, since, 


$$
\begin{aligned}
& \int_{\Omega}\left(a\left(x, D u_{\varepsilon}\right)-a(x, D u)\right) D\left(u_{\varepsilon}-u\right) \mathrm{d} x \\
& \quad=\int_{\Omega} a\left(x, D u_{\varepsilon}\right) D\left(u_{\varepsilon}-u\right) \mathrm{d} x-\int_{\Omega} a(x, D u) D\left(u_{\varepsilon}-u\right) \mathrm{d} x
\end{aligned}
$$

we have,

$$
\lim _{\varepsilon \rightarrow 0} \int_{\Omega}\left(a\left(x, D u_{\varepsilon}\right)-a(x, D u)\right) D\left(u_{\varepsilon}-u\right) \mathrm{d} x=0
$$

from a lemma of [6] it implies that,

$$
\lim _{\varepsilon \rightarrow 0}\left\|D\left(u_{\varepsilon}-u\right)\right\|_{p}=0
$$

This implies that we can pass to the limit in the equation satisfied by $u_{\varepsilon}$, and we get $u=A(v)$. Consequently the whole sequence $\left(u_{\varepsilon}\right)$ converges to $u$ and finally it proves that $A$ is continuous.

- With same arguments we can prove that $A(E)$ is precompact. Indeed if $\left(u_{\varepsilon}\right)$ is a bounded sequence in $A(E)$ then $u_{\varepsilon}=A\left(v_{\varepsilon}\right)$ with $\left(v_{\varepsilon}\right)$ or a subsequence is such that,

$$
v_{\varepsilon} \rightarrow v \text { a.e. } \quad \text { in } \Omega \text { and } L^{p}(\Omega) \text { strong }
$$

and we deduce like previously that,

$$
u_{\varepsilon} \rightarrow u \quad \text { in } W_{0}^{1, p}(\Omega) \text { strong. }
$$

End of the proof of Theorem 1.1.

Let $\mu \in M_{B}(\Omega)$ such that $|\mu|(\Omega)=1$ and $m \in \mathbb{R}$, then $m \mu$ can be decomposed as,

$$
m \mu=f-\operatorname{div} g+\lambda^{+}-\lambda^{-} .
$$

Let $\left(\mu_{n}\right)$ a sequence of measures in $M_{B}(\Omega)$ such that,

$$
\mu_{n}=f_{n}-\operatorname{div} g+\lambda_{n}^{\oplus}-\lambda_{n}^{\ominus}
$$

with,

$$
f_{n} \in L^{p^{\prime}}(\Omega) \text { and }\left(f_{n}\right) \text { converges to } f \text { weakly in } L^{1}(\Omega),
$$

$\lambda_{n}^{\oplus}$ is a sequence of nonnegative functions in $L^{p^{\prime}}(\Omega)$ that

converges to $\mu_{s}^{+}$in the narrow topology of measures,

$\lambda_{n}^{\ominus}$ is a sequence of nonnegative functions in $L^{p^{\prime}}(\Omega)$ that converges to $\mu_{s}^{-}$in the narrow topology of measures, 


$$
\left|\mu_{n}\right|(\Omega) \leqslant m,
$$

then there exists a solution $u_{n}$ of the corresponding Eq. (3.1) which satisfies

$$
\left\|u_{n}\right\|_{\gamma q^{\prime}} \leqslant Y
$$

Observe that in (3.1) the right-hand side is bounded in $M_{B}(\Omega)$, then it is proven in [8] that we can extract a subsequence which converges in measure and a.e. in $\Omega$ to a measurable function $u$ which is finite almost everywhere. Moreover since the right-hand side in (3.1) is bounded in $M_{B}(\Omega)$, from Lemma 2.1 we have, if $q \neq 1$, with a small $\delta$

$$
\left\|u_{n}\right\|_{\gamma q^{\prime}+\delta} \leqslant C
$$

where $C$ is a constant which does not depend on $n$. We deduce that $\left(u_{n}^{\gamma q^{\prime}}\right)$ converges to $\left(u^{\gamma q^{\prime}}\right)$ in $L^{1}(\Omega)$ strong (see [3]). Moreover, we have,

$$
\left\|f(x)\left|u_{n}\right|^{\gamma}-f(x)|u|^{\gamma}\right\|_{L^{1}(\Omega)} \leqslant\|f\|_{q}\left(\int_{\Omega}\left(\left|u_{n}\right|^{\gamma}-|u|^{\gamma}\right)^{q^{\prime}}\right)^{1 / q^{\prime}}
$$

but, $\left(\left|u_{n}\right|^{\gamma}-|u|^{\gamma}\right)^{q^{\prime}}$ tends to 0 a.e. in $\Omega$ and,

$$
\left(\left|u_{n}\right|^{\gamma}-|u|^{\gamma}\right)^{q^{\prime}} \leqslant 2^{q^{\prime}-1}\left|u_{n}\right|^{\gamma q^{\prime}}+2^{q^{\prime}-1}|u|^{\gamma q^{\prime}} .
$$

The right-hand side converges in $L^{1}(\Omega)$ strong. Then by Vitali Lemma and (3.8), we deduce that,

$$
f(x)\left|u_{n}\right|^{\gamma} \text { tends to } f(x)|u|^{\gamma} \text { in } L^{1}(\Omega) \text { strong. }
$$

We assert again that $h_{n}\left(x, u_{n}\right)$ converges a.e. in $\Omega$ to $h(x, u)$ and by (1.8) and (3.9), we deduce that $h_{n}\left(x, u_{n}\right)$ converges to $h(x, u)$ in $L^{1}(\Omega)$ strong. The same conclusion holds when $q=1$. So $f_{n}+h_{n}\left(x, u_{n}\right)$ converges in $L^{1}(\Omega)$ weak, and with the additionnal assumptions (3.5), (3.6) on $\lambda_{n}^{\ominus}$ and $\lambda_{n}^{\oplus}$ we can apply Theorem 3.2 of [8] and conclude that $u$ is a renormalized solution of (3.1).

\section{Acknowledgement}

The author thanks Francois Murat for fruitful discussions in Bourges.

\section{REFERENCES}

[1] Adams D.R., Pierre M., Capacitary strong type estimates in semilinear problems, Ann. Inst. Fourier, Grenoble 41 (1991) 117-135.

[2] Baras P., Pierre M., Critère d'existence de solutions positives pour des équations semilinéaires non monotones, Ann. Inst. H. Poincaré, Analyse Non Linéaire 2 (1985) 185-212.

[3] Benilan P., Boccardo L., Gallouët T., Gariepy R., Pierre M., Vazquez J.L., An $L^{1}$ theory of existence uniqueness of nonlinear elliptic equations, Ann. Scuola Norm. Sup. Pisa Cl.

Sci. 22 (1995) 241-273. 
[4] Brezis H., Cabré X., Some simple nonlinear PDE's without solutions, Bolletino U.M.I. 1-B (1998) 223-262.

[5] Boccardo L., Gallouët T., Orsina L., Existence and uniqueness of entropy solutions for nonlinear elliptic equations with measure data, Ann. Inst. H. Poincaré Anal. Non Linéaire 13 (1996) 539-551.

[6] Boccardo L., Murat F., Puel J.P., Existence of bounded solutions for nonlinear elliptic unilateral problem, Ann. di Mat. Pura ed Appl. 152 (1988) 183-196.

[7] Dal Maso G., Murat F., Orsina L., Prignet A., Definition and existence of renormalized solutions of elliptic equations with general measure data, C. R. Acad. Sci. Paris Série I 325 (1997) 481-486.

[8] Dal Maso G., Murat F., Orsina L., Prignet A., Renormalized solutions of elliptic equations with general measure data, Ann. Scuol. Norm. Pisa (4) XXVIII (1999) 741-808.

[9] Ferone V., Murat F., Nonlinear problems having natural growth in the gradient: an existence result when the source term is small, to appear.

[10] Fukushima M., Sato K., Taniguchi S., On the closable part of pre-Dirichlet forms and the fine support of the underlying measures, Osaka J. Math. 28 (1991) 517-535.

[11] Kalton N.J., Verbitsky E., Nonlinear equations and weighted norm inequalities, Trans. Amer. Math. Soc. 351 (9) 3441-3497.

[12] Grenon N., Existence and comparison results quasilinear elliptic equations with quadratic growth in the gradient, J. Differential Equations, to appear.

[13] Grenon N., $L^{r}$ estimates for degenerate elliptic problems, Pot. Anal., to appear.

[14] Grenon-Isselkou N., Mossino J., Existence de solutions bornées pour certaines équations elliptiques quasilinéaires, C. R. Acad. Sci. 321 (1995) 51-56.

[15] Orsina L., Solvability of linear and semilinear eigenvalue problems with $L^{1}$ data, Rend. Sem. Mat. Univ. Padova 90 (1993).

[16] Stampacchia G., Le problème de Dirichlet pour les équations elliptiques du second ordre à coefficients discontinus, Ann. Inst. Fourier (Grenoble) 15 (1965) 189-258.

[17] Talenti G., Linear elliptic P.D.E.'s: Level sets, rearrangements and a priori estimates of solutions, Boll. U.M.I. (6) 4-B (1985) 917-949. 